\title{
Homogeneity in traditional knowledge and cultural importance of wild edible plants in Kishtwar - a Himalayan district in North- West Himalaya
}

\author{
Sajan Thakur and Harish Chander Dutt ${ }^{1}$ \\ Ecological Engineering Laboratory, Department of Botany, University of Jammu, Jammu - 180006, \\ Jammu \& Kashmir-, India \\ ${ }^{1}$ Communicating author, e-mail: hcdutt@rediffmail.com; sajan0007thakur@gmail.com
}

[Received 16.11.2020; Revised 26.12.2020; Accepted 27.12.2020; Published 31.12.2020]

\begin{abstract}
Wild edible plants play an important role in the rural life. A total of 130 such plant species are documented from district Kishtwar of UT Jammu and Kashmir. During the survey 78 informants were interviewed in the region and data collected were analyzed for homogeneity in traditional knowledge and cultural importance using Factor informant consensus (Fic) and Cultural importance index $(C I)$. Higher Fic more than 0.90 describes more homogeneity of knowledge among the informants regarding the use of wild edible plants in the study area. Ferula jaeschkeana, Elwendia persica, Diplazium esculentum, Berberis lycium and Juglans regia are identified as culturally most important vegetable and fruit species in the study area. Rosaceae contributed maximum species in the traditional culture of the inhabitants of Kishtwar as wild edible species.
\end{abstract}

Key words: Wild food, Cultural importance index, Factor informant consensus, Kishtwar.

\section{INTRODUCTION}

The uncultivated plant species that are accessed from natural habitats and used as food are known as wild edible plants (Beluhan \& Ranogajec 2010). These are collected traditionally from the forests, waste lands, roadsides and banks of streams and rivers. People of developing countries generally reside in local villages, ethnic communities and tribal villages. Most of them have a long history of use of such plant species for driving their livelihood (Schippmann et al. 2002). People are using these plant species since time immemorial and may have utilized more than 7000 such plants so far, but many more such food species are not documented till date (Grivetti and Ogle 2000; Mohan Ram 2000).

According to a report of Food and Agricultural Organization (FAO), about one billion people of the world use wild plants as the source of their diet (Burlingame 2000). These plant species not only provide nutrition to many malnourished people but also act as source of food during food shortage and famine periods (Hussain et al. 2009). In India wild edible plants have achieved an important place in the ethnic and traditional cultures. In this context, various workers have recorded the use of wild edible plants from different parts of Himalaya (Jain 1964; Negi, 1988; Haridasan et al. 1990; Pundir \& Singh 2002; Kulkarni et al. 2003; Kumer 2003; Sundriyal et al., 2004; Kayang, 2007; Rashid 2008; Rathore 2009; Bhatia et al. 2018; Singh et al. 2019; Pandita \& Dutt 2018; Thakur et al. 2020a) and other parts of India (Binu, 2010; Vizhi \& Lohidas, 2020).

Kishtwar - a hilly district of UT of Jammu and Kashmir in north-west Himalaya is characterized by temperate to alpine conditions. Due to tough terrain, geography and peculiar cold climate during winter various villages of the district remain cut off from the major cities of the province for 3-4 months. The instinct, need, observations, trials and errors along with 
experiences gained through day-to-day human life in such areas has led to the use of the wild edible plants. The people are familiar with the wild natural resources and derive majority of their livelihood from them. The major occupation of the people is agriculture and livestock rearing. During the favorable climatic conditions these people also derive their earnings by selling the wild produce to the nearby markets. Among the wild resources the wild edible plants are the major chunk for utilization and generation of economy by the inhabitants of the villages of the district. The acquired traditional knowledge is transferred from one generation to the other by these people through oral dialogue. In many other regions of the world this age-old traditional knowledge is seen to be getting faded away in the light of developmental activities, mass migration from rural to urban areas, modern education, changing cultures and traditions, decline in natural resources and attraction towards western ways of life (Luczaj et al. 2013; Rao et al. 2015; Reyes-Garcia et al. 2015; Bhatia et al. 2015). Therefore, it is important to collect, document, preserve and disseminate this age-old traditional knowledge for the betterment of the society. The present study was undertaken to document and evaluate the cultural significance and consensus among the local people with respect to usage of wild edible plants in Kishtwar.

\section{MATERIAL AND METHODS}

Situated between $31^{\circ} 06^{\prime}$ to $32^{\circ} 18^{\prime} \mathrm{N}$ latitude and $75^{\circ} 24^{\prime}$ to $76^{\circ} 42^{\prime} \mathrm{E}$ longitude Kishtwar district is spreadover in an area of $7737 \mathrm{sq}$. Kms. It is the largest district of Union Territory of Jammu and Kashmir (J\&K) with altitude ranging from 900 to 6300 meters above msl. Its boundaries touch Doda district in south-west, Anantnag district of Kashmir in west, Ladakh in north-east and Himachal in south-east directions (Figure 1). It is the largest and least popu-

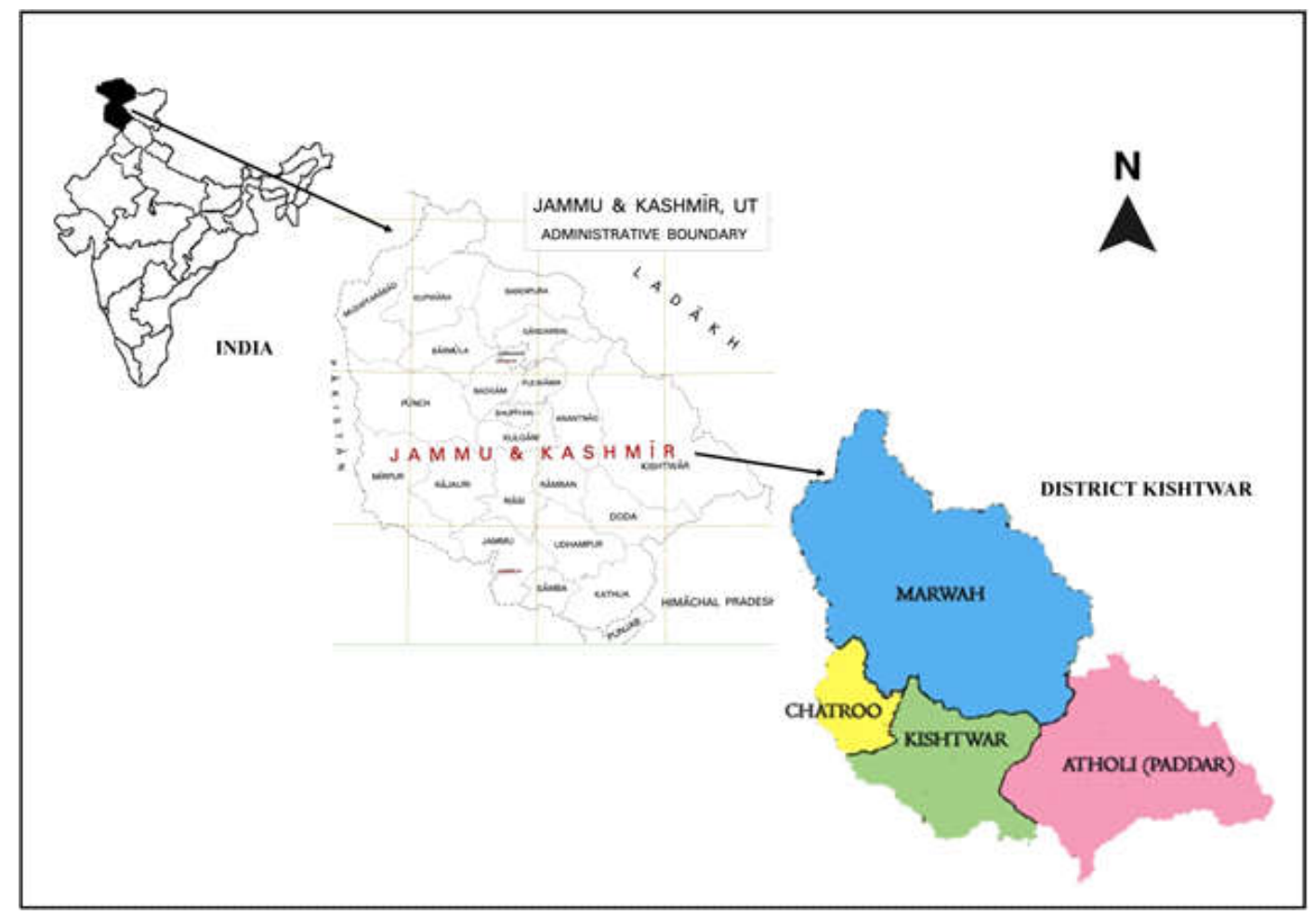

Figure 1. Map of the Study area 
lous district of UT of J\&K. This mountainous area is characterized by temperate vegetation dominated by Pinus wallichiana AB Jacks. (Pinaceae) at low and Cedrus deodara (Roxb. ex D.Don) G.Don (Pinaceae) at high elevations. Agricultural system is mostly dependent on seasonal rains and therefore only rain-fed crops such as maize, wheat, barley, oats and mustard are cultivated by local inhabitants in the region. Among fruit trees apple, apricot, pear, quince and walnut are the main horticultural crops. The region is also known for cultivation of saffron (Crocus sativus L.), and is considered as the second largest region of saffron production after Pampore in Jammu and Kashmir, India (Thakur et al. 2020b).

Various systematic and extensive ethno-botanical surveys were conducted in different areas of Kishtwar district during March 2019 to October 2020 for the collection of information on wild edible plant species being used by the local people. The informants were selected randomly and information was gathered by conducting personnel interviews and group discussions with the informants in their local language on the indigenous uses of wild flora as food. A total of 78 informants ( 33 male and 45 female) between the age group 15-75 years were interviewed with a semi structured questionnaire. The information collected included common wild edible plant species, local names, plant part used, and recipe prepared. The plant species were identified from the herbaria of the Department of Botany, University of Jammu, Jammu, and Indian Institute of Integrative Medicine, Jammu, and also with the help of various regional floras (Sharma \& Kachroo, 1983; Swami \& Gupta, 1998). The final list of the plants was prepared following the online database "Plants of the world online- www.plantsoftheworldonline.org" for the correct botanical nomenclature.

The data gathered through questionnaire and interviews were analyzed quantitatively using two ethnobotanical indices as follows:

Factor informant consensus (Fic): This index is used to test the homogeneity of knowledge about the wild edible plants among the informants and is calculated according to Heinrich $e t a l$. (1998):

$$
\text { Fic }=\mathrm{Nur}^{-} \mathrm{Nt} / \mathrm{Nur}^{-} 1
$$

Where, nur refers to the number of use-reports for a particular use category and $n t$ refers to the number of taxa used for a particular use category by all the informants. Fic values are low (near 0 ) if plants are chosen randomly or if there is no exchange of information about their use among informants, and approach one (1) when there is a well-defined selection criterion in the community and/or if information is exchanged between informants (Gazzaneo et al. 2005; Sharma et al. 2012).

Cultural importance index (CI): This index determines the cultural importance and significance of a species as source of wild food and is calculated after Tardio and Pardo (2008) as below:

$$
C I=\sum_{u=u 1}^{u-N C} \cdot \sum_{i=i 1}^{i N} \text { URui } / N
$$

CI index can also be seen as the sum of the proportion of informants that mention each species use. This additive index takes into account not only the spread of the use (number of informants) for each species but also diversity in usage. The theoretical maximum value of the index is the total number of different use-categories (NC), reached in the unlikely case that all the informants would mention the use of the species in all the usecategories considered in a survey. 


\section{RESULTS}

A total of 130 plant species belonging to 104 genera and 52 families served as wild food in Kishtwar district (Table 1; Figure 2). Most represented plant genus was Rubus with 6 species followed by Allium and Rumex with 3 species each. Maximum species are contributed by Rosaceae (14 species) followed by Apiaceae and Polygonaceae ( 9 species each), Fabaceae (8 species) and Asteraceae and Brassicaceae (7 species each). Herbaceous species ( 85 species) is a life form which contributed maximum number followed by shrubs (24 species), trees (19 species) and climbers (2 species) (Figure 3 ) as wild plant species. People in Kishtwar consume leaves of 49 species fruits of 42 species, root and complete shoot of 10 each, seeds of 10 , flowers of 6 , bulb of 4 , tuber of 3 , bark of 2 and rhizome of 1 plant species (Figure 4).

Table 1. Edible usage and cultural index of wild edible plants of district Kishtwar.

\begin{tabular}{|c|c|c|c|c|c|}
\hline Botanical name [Family] & $\begin{array}{l}\text { Local } \\
\text { name }\end{array}$ & Habit & $\begin{array}{l}\text { Part } \\
\text { used }\end{array}$ & Uses & $\mathbf{C I}$ \\
\hline $\begin{array}{l}\text { Aesculus indica (Wall. ex } \\
\text { Cambess.) Hook. }\end{array}$ & Bankhod & $\mathrm{T}$ & Fruit & $\begin{array}{l}\text { Nuts are processed into flour } \\
\text { and mixed with Barley flour to } \\
\text { make traditional bread called } \\
\text { "Khamerey" (12)* }\end{array}$ & 0.15 \\
\hline $\begin{array}{l}\text { Allium atropurpureum Waldst. } \\
\text { \& Kit. [Amaryllidaceae] }\end{array}$ & Wanpraan & $\mathrm{H}$ & Leaf & $\begin{array}{l}\text { Leaves are used as spice in } \\
\text { vegetable }(10)\end{array}$ & 0.13 \\
\hline $\begin{array}{l}\text { Allium humile Kunth } \\
\text { [Amaryllidaceae] }\end{array}$ & $\begin{array}{l}\text { Jangli pyaz, } \\
\text { Kuthe, } \\
\text { Shauth }\end{array}$ & $\mathrm{H}$ & $\begin{array}{l}\text { Leaf, } \\
\text { Bulb }\end{array}$ & $\begin{array}{l}\text { Leaves are used for making } \\
\text { vegetable, either with other } \\
\text { vegetables or separately (18). } \\
\text { Bulb is used as spices (15). }\end{array}$ & 0.42 \\
\hline $\begin{array}{l}\text { Allium roylei Stearn } \\
\text { [Amaryllidaceae] }\end{array}$ & Bazun & $\mathrm{H}$ & Leaf & $\begin{array}{l}\text { Dried leaves used as spices } \\
\text { (28) }\end{array}$ & 0.36 \\
\hline $\begin{array}{l}\text { Amaranthus cruentus } \mathrm{L} \text {. } \\
\text { [Amaranthaceae] }\end{array}$ & Seol & $\mathrm{H}$ & $\begin{array}{l}\text { Seed, } \\
\text { Leaf }\end{array}$ & $\begin{array}{l}\text { Seeds are cooked as pudding } \\
\text { (Kheer) (13) and leaves are } \\
\text { eaten as vegetable (23). }\end{array}$ & 0.46 \\
\hline $\begin{array}{l}\text { Amaranthus viridis } \mathrm{L} \text {. } \\
\text { [Amaranthaceae] }\end{array}$ & Zerar & $\mathrm{H}$ & $\begin{array}{l}\text { Leaf, } \\
\text { Shoot }\end{array}$ & $\begin{array}{l}\text { Young shoots and leaves are } \\
\text { cooked as vegetable (26). }\end{array}$ & 0.33 \\
\hline $\begin{array}{l}\text { Anethum graveolens } \mathrm{L} . \\
\text { [Apiaceae] }\end{array}$ & Soa & $\mathrm{H}$ & Seed & $\begin{array}{l}\text { Seeds are used as spice and } \\
\text { condiment (12). }\end{array}$ & 0.15 \\
\hline $\begin{array}{l}\text { Angelica glauca Edgew. } \\
\text { [Apiaceae] }\end{array}$ & Chorai & $\mathrm{H}$ & Root & $\begin{array}{l}\text { Roots are used as spice and } \\
\text { condiments (14). }\end{array}$ & 0.18 \\
\hline $\begin{array}{l}\text { Apteranthes tuberculata } \\
\text { (N.E.Br.) Meve \& Leide } \\
\text { [Apocynaceae] }\end{array}$ & Charungli & S & Tuber & $\begin{array}{l}\text { Tubers are cooked and also } \\
\text { pickled for winter } \\
\text { consumption (25). }\end{array}$ & 0.32 \\
\hline $\begin{array}{l}\text { Arenaria nevadensis Boiss. \& } \\
\text { Reut. [Caryophyllaceae] }\end{array}$ & Chiki & $\mathrm{H}$ & Leaf & $\begin{array}{l}\text { Tender leaves are cooked as } \\
\text { vegetables (18). }\end{array}$ & 0.23 \\
\hline $\begin{array}{l}\text { Arisaema propinquum Schott } \\
\text { [Araceae] }\end{array}$ & Jamushung & $\mathrm{H}$ & Bulb & $\begin{array}{l}\text { Bulbs used for preparation of } \\
\text { local wine }(9) *\end{array}$ & 0.12 \\
\hline $\begin{array}{l}\text { Arnebia euchroma (Royle ex } \\
\text { Benth) I.M.Jonst. [Boraginaceae] }\end{array}$ & Aamokh & $\mathrm{H}$ & Root & $\begin{array}{l}\text { Roots used to make soups and } \\
\text { stews }(20) *\end{array}$ & 0.26 \\
\hline $\begin{array}{l}\text { Arnebia guttata Bunge } \\
\text { [Boraginaceae] }\end{array}$ & Demokh & $\mathrm{H}$ & Root & $\begin{array}{l}\text { Roots used to make soups and } \\
\text { stews (12). * }\end{array}$ & 0.15 \\
\hline $\begin{array}{l}\text { Astragalus chlorostachys } \\
\text { Lindl. [Fabaceae] }\end{array}$ & $\begin{array}{l}\text { Aiste- } \\
\text { Kachh }\end{array}$ & $\mathrm{H}$ & Fruit & Fruits eaten raw (21). & 0.27 \\
\hline $\begin{array}{l}\text { Berberis aristata DC. } \\
\text { [Berberidaceae] }\end{array}$ & Khumlai & $\mathrm{S}$ & Fruit & Fruit is edible (38). & 0.49 \\
\hline
\end{tabular}


Sajan Thakur \& Harish Chander Dutt 281

\begin{tabular}{|c|c|c|c|c|c|}
\hline Botanical name [Family] & $\begin{array}{l}\text { Local } \\
\text { name }\end{array}$ & Habit & $\begin{array}{l}\text { Part } \\
\text { used }\end{array}$ & Uses & $\mathrm{CI}$ \\
\hline $\begin{array}{l}\text { Berberis lycium Royle } \\
\text { [Berberidaceae] }\end{array}$ & Khumlai & S & Fruit & $\begin{array}{l}\text { Fruits are edible once turned } \\
\text { black (40). }\end{array}$ & 0.51 \\
\hline $\begin{array}{l}\text { Bergenia ciliata (Haw.) Sternb. } \\
\text { [Saxifragaceae] }\end{array}$ & Lau & $\mathrm{H}$ & Leaf & $\begin{array}{l}\text { Leaves are used for making } \\
\text { fritters (Pakora) (14). }\end{array}$ & 0.18 \\
\hline $\begin{array}{l}\text { Betula utilis D.Don } \\
\text { [Betulaceae] }\end{array}$ & Bhojpatr, & $\mathrm{T}$ & Bark & $\begin{array}{l}\text { Powder of bark used for } \\
\text { preparing tea (11). }{ }^{*}\end{array}$ & 0.14 \\
\hline $\begin{array}{l}\text { Bistorta amplexicaulis } \\
\text { (D.Don) Greene [Polygonaceae] }\end{array}$ & Remu & $\mathrm{H}$ & Root & $\begin{array}{l}\text { Roots used as a tea substitute } \\
\text { (7). }\end{array}$ & 0.09 \\
\hline $\begin{array}{l}\text { Bupleurum falcatum } \mathrm{L} . \\
\text { [Apiaceae] }\end{array}$ & Jeeraghas & $\mathrm{H}$ & Seed & Seeds are used as spice (17). & 0.22 \\
\hline $\begin{array}{l}\text { Cannabis sativa L. } \\
\text { [Cannabaceae] }\end{array}$ & Bhang & $\mathrm{H}$ & Leaf & $\begin{array}{l}\text { Leaves are used in sedative } \\
\text { drinks (26). }\end{array}$ & 0.33 \\
\hline $\begin{array}{l}\text { Capsella bursa-pastoris (L.) } \\
\text { Medik. [Brassicaceae] }\end{array}$ & Khathkram & $\mathrm{H}$ & Leaf & $\begin{array}{l}\text { Leaves are cooked as vegetable } \\
\text { (13). }\end{array}$ & 0.17 \\
\hline $\begin{array}{l}\text { Cardamine hirsuta L. } \\
\text { [Brassicaceae] }\end{array}$ & Kukdi & $\mathrm{H}$ & Leaf & $\begin{array}{l}\text { Tender leaves are used for } \\
\text { making vegetables (9). }\end{array}$ & 0.12 \\
\hline Carum carvi L. [ Apiaceae] & $\begin{array}{l}\text { Makoh- } \\
\text { zeera }\end{array}$ & $\mathrm{H}$ & Seed & Seeds are used as spices (20). & 0.26 \\
\hline $\begin{array}{l}\text { Cassiope fastigiata (Wall.) } \\
\text { D.Don [Ericaceae] }\end{array}$ & Tolo & $\mathrm{H}$ & Flower & $\begin{array}{l}\text { Dried flowers are used for } \\
\text { making tea (8). }\end{array}$ & 0.1 \\
\hline $\begin{array}{l}\text { Celosia argentea } \mathrm{L} . \\
\text { [Amaranthaceae] }\end{array}$ & - & $\mathrm{H}$ & Leaf & $\begin{array}{l}\text { Leaves are cooked as vegetable } \\
\text { (16). }\end{array}$ & 0.21 \\
\hline $\begin{array}{l}\text { Celtis australis } \mathrm{L} . \\
\text { [Cannabaceae] }\end{array}$ & Khidik & $\mathrm{T}$ & $\begin{array}{l}\text { Fruit, } \\
\text { Seed }\end{array}$ & $\begin{array}{l}\text { Ripe fruits eaten raw (10) and } \\
\text { ground to prepare flour (14) }\end{array}$ & 0.31 \\
\hline $\begin{array}{l}\text { Centaurea iberica Trevir. ex } \\
\text { Spreng. [Asteraceae] }\end{array}$ & Krotzkond & $\mathrm{H}$ & Leaf & $\begin{array}{l}\text { Tender leaves are eaten as } \\
\text { vegetable (11). }\end{array}$ & 0.14 \\
\hline $\begin{array}{l}\text { Chaerophyllum reflexum } \\
\text { Lindl. [Apiaceae] }\end{array}$ & Nyoch & $\mathrm{H}$ & Root & $\begin{array}{l}\text { Roots are eaten as salad (8) } \\
\text { and vegetable (10). }\end{array}$ & 0.23 \\
\hline $\begin{array}{l}\text { Chaerophyllum villosum } \\
\text { Wall. ex DC. [Apiaceae] }\end{array}$ & Nyoch & $\mathrm{H}$ & Root & $\begin{array}{l}\text { Roots are eaten as salad (6) } \\
\text { and vegetable (6). }\end{array}$ & 0.15 \\
\hline $\begin{array}{l}\text { Chenopodium album } \mathrm{L} \text {. } \\
\text { [Amaranthaceae] }\end{array}$ & Bajarbhang & $\mathrm{H}$ & Leaf & $\begin{array}{l}\text { Leaves are cooked as vegetable } \\
\text { (15). }\end{array}$ & 0.19 \\
\hline $\begin{array}{l}\text { Cicer songaricum Stephan ex } \\
\text { DC. [Fabaceae] }\end{array}$ & Seri-jriboo & $\mathrm{H}$ & Fruit & $\begin{array}{l}\text { Unripe legumes are cooked as } \\
\text { vegetable (7). }\end{array}$ & 0.09 \\
\hline $\begin{array}{l}\text { Cichorium intybus } \mathrm{L} \text {. } \\
\text { [Asteraceae] }\end{array}$ & Kasini & $\mathrm{H}$ & Shoot & $\begin{array}{l}\text { Tender plants are cooked as } \\
\text { vegetable (8). }\end{array}$ & 0.1 \\
\hline $\begin{array}{l}\text { Cirsium arvense (L.) Scop. } \\
\text { [Asteraceae] }\end{array}$ & Kandmool & $\mathrm{H}$ & Root & Roots are eaten raw (5). & 0.06 \\
\hline $\begin{array}{l}\text { Codonopsis ovata Benth. } \\
\text { [Campanulaceae] }\end{array}$ & Ludut & $\mathrm{H}$ & Root & $\begin{array}{l}\text { Root are cooked as vegetable } \\
\text { (6). }\end{array}$ & 0.08 \\
\hline $\begin{array}{l}\text { Colocasia esculenta }(\mathrm{L} .) \text { Schott } \\
\text { [Araceae] }\end{array}$ & Alvathur & $\mathrm{H}$ & Leaf & $\begin{array}{l}\text { Leaves are cooked as vegetable } \\
(10) .\end{array}$ & 0.13 \\
\hline $\begin{array}{l}\text { Commelina benghalensis } \mathrm{L} \text {. } \\
\text { [Commelinaceae] }\end{array}$ & Chura & $\mathrm{H}$ & Shoot & $\begin{array}{l}\text { Plant used as vegetable (8) and } \\
\text { to make fritters (8). }\end{array}$ & 0.21 \\
\hline $\begin{array}{l}\text { Coriaria nepalensis Wall. } \\
\text { [Coriariaceae] }\end{array}$ & Hang & S & Fruit & Fruits edible (6). & 0.08 \\
\hline $\begin{array}{l}\text { Cornus macrophylla Wall. } \\
\text { [Cornaceae] }\end{array}$ & Khagsoo & $\mathrm{T}$ & Fruit & Ripe fruits are eaten raw (12). & 0.15 \\
\hline
\end{tabular}




\begin{tabular}{|c|c|c|c|c|c|}
\hline Botanical name [Family] & $\begin{array}{l}\text { Local } \\
\text { name }\end{array}$ & Habit & $\begin{array}{l}\text { Part } \\
\text { used }\end{array}$ & Uses & CI \\
\hline $\begin{array}{l}\text { Corylus avellana L. } \\
\text { [Betulaceae] }\end{array}$ & Thanki & $\mathrm{T}$ & Fruit & Kernels are edible (23). & 0.29 \\
\hline $\begin{array}{l}\text { Corylus jacquemontii Decne. } \\
\text { [Betulaceae] }\end{array}$ & Thanki & $\mathrm{T}$ & Fruit & Kernels are edible (26). & 0.33 \\
\hline $\begin{array}{l}\text { Cotoneaster microphyllus } \\
\text { Wall. ex Lindl. [Rosaceae] }\end{array}$ & Reunsh & $\mathrm{S}$ & Fruit & Ripe fruits are eaten (18). & 0.23 \\
\hline $\begin{array}{l}\text { Crataegus songarica K.Koch } \\
\text { [Rosaceae] }\end{array}$ & Khring & $\mathrm{T}$ & Fruit & Fruit is edible (13). & 0.17 \\
\hline $\begin{array}{l}\text { Crucihimalaya himalaica } \\
\text { (Edgew.) Al-Shehbaz, O'Kane \& } \\
\text { R.A.Price [Brassicaceae] }\end{array}$ & Sikiang & $\mathrm{H}$ & Leaf & $\begin{array}{l}\text { Tender leaves are washed, cut, } \\
\text { boiled and fried to make } \\
\text { vegetable (6). }\end{array}$ & 0.08 \\
\hline $\begin{array}{l}\text { Daphne gnidioides Jaub. \& } \\
\text { Spach [Thymelaeaceae] }\end{array}$ & Chikoh & $\mathrm{S}$ & Fruit & $\begin{array}{l}\text { Ripe fruits are used for making } \\
\text { local wine (9).* }\end{array}$ & 0.12 \\
\hline $\begin{array}{l}\text { Debregeasia saeneb (Forssk.) } \\
\text { Hepper \& J.R.I.Wood } \\
\text { [Urticaceae] }\end{array}$ & Tushiari, & $\mathrm{S}$ & Fruit & Ripe fruits are eaten raw (8). & 0.1 \\
\hline $\begin{array}{l}\text { Dioscorea deltoidea Wall. ex } \\
\text { Griseb. [Dioscoreaceae] }\end{array}$ & Shingli & $\mathrm{C}$ & Tuber & $\begin{array}{l}\text { Tubers cut into slices, tied in } \\
\text { cloth and kept in running } \\
\text { water in streams or riverbeds } \\
\text { overnight; these are then } \\
\text { boiled like potato and eaten (6) } \\
\text { *; tubers sometimes roasted in } \\
\text { low fire to eat }(9)\end{array}$ & 0.19 \\
\hline $\begin{array}{l}\text { Diospyros lotus L. } \\
\text { [Ebenaceae] }\end{array}$ & Amlok & $\mathrm{T}$ & Fruit & Fruits eaten raw (17). & 0.22 \\
\hline $\begin{array}{l}\text { Diplazium esculentum (Retz.) } \\
\text { Sw. [Aspleniaceae] }\end{array}$ & Vani/Dhed & $\mathrm{H}$ & Leaf & $\begin{array}{l}\text { Fronds cooked as vegetable } \\
\text { (46). }\end{array}$ & 0.59 \\
\hline $\begin{array}{l}\text { Diplazium maximum (D.Don) } \\
\text { C.Chr. [Aspleniaceae] }\end{array}$ & Kakhish & $\mathrm{H}$ & Leaf & $\begin{array}{l}\text { Young leaves and fronds } \\
\text { cooked as vegetable (31). }\end{array}$ & 0.4 \\
\hline $\begin{array}{l}\text { Dipsacus inermis Wall. } \\
\text { [Caprifoliaceae] }\end{array}$ & Wapal hakh & $\mathrm{H}$ & Leaf & $\begin{array}{l}\text { Leaves cooked as vegetable } \\
(15) .\end{array}$ & 0.19 \\
\hline $\begin{array}{l}\text { Elaeagnus umbellata Thunb. } \\
\text { [Elaeagnaceae] }\end{array}$ & Goain & $\mathrm{S}$ & Fruit & Fruits edible (10) & 0.13 \\
\hline $\begin{array}{l}\text { Elwendia persica (Boiss.) } \\
\text { Pimenov \& Kljuykov [Apiaceae] }\end{array}$ & $\begin{array}{l}\text { Krishun } \\
\text { Zeur }\end{array}$ & $\mathrm{H}$ & Seed & $\begin{array}{l}\text { Seeds used as spice and } \\
\text { condiment (46) }\end{array}$ & 0.59 \\
\hline $\begin{array}{l}\text { Ephedra gerardiana Wall. ex } \\
\text { Klotzsch \& Garcke } \\
\text { [Ephedraceae] }\end{array}$ & Cheldum & $\mathrm{H}$ & Fruit & $\begin{array}{l}\text { Ripe fruits are sweet and } \\
\text { consumed by Shepherds when } \\
\text { they go to forest with their } \\
\text { sheep (21). }\end{array}$ & 0.27 \\
\hline $\begin{array}{l}\text { Equisetum arvens L. } \\
\text { [Equisetaceae] }\end{array}$ & Paccu & $\mathrm{H}$ & Shoot & $\begin{array}{l}\text { Young shoots are consumed } \\
\text { raw (13). }\end{array}$ & 0.17 \\
\hline $\begin{array}{l}\text { Eremurus himalaicus Baker } \\
\text { [Asphodelaceae] }\end{array}$ & Hulla & $\mathrm{H}$ & Leaf & $\begin{array}{l}\text { Leaves cooked as vegetable } \\
(14) .\end{array}$ & 0.14 \\
\hline $\begin{array}{l}\text { Erodium tibetanum Edgew. \& } \\
\text { Hook.f. [Geraniaceae] }\end{array}$ & Tikziang & $\mathrm{H}$ & Leaf & $\begin{array}{l}\text { Tender leaves are used for } \\
\text { making vegetable (9). }\end{array}$ & 0.12 \\
\hline $\begin{array}{l}\text { Ferula jaeschkeana Vatke } \\
\text { [Apiaceae] }\end{array}$ & Hing & $\mathrm{H}$ & Leaf & $\begin{array}{l}\text { Tender leaves and shoots are } \\
\text { used as vegetable (18). Latex is } \\
\text { used as spice (32). }\end{array}$ & 0.64 \\
\hline
\end{tabular}


Sajan Thakur \& Harish Chander Dutt 283

\begin{tabular}{|c|c|c|c|c|c|}
\hline Botanical name [Family] & $\begin{array}{l}\text { Local } \\
\text { name }\end{array}$ & Habit & $\begin{array}{l}\text { Part } \\
\text { used }\end{array}$ & Uses & $\mathbf{C I}$ \\
\hline $\begin{array}{l}\text { Ficus palmata Forssk. } \\
\text { [Moraceae] }\end{array}$ & Fag & $\mathrm{T}$ & Fruit & Fruits are edible (28). & 0.36 \\
\hline $\begin{array}{l}\text { Fragaria nubicola (Lindl. ex } \\
\text { Hook.f.) Lacaita [Rosaceae] }\end{array}$ & Ingdach & $\mathrm{H}$ & Fruit & $\begin{array}{l}\text { Fruit is edible (23), roots used } \\
\text { as tea substitute (10). }\end{array}$ & 0.44 \\
\hline $\begin{array}{l}\text { Fritillatia cirthosa D.Don } \\
\text { [Liliaceae] }\end{array}$ & Sheethkar & $\mathrm{H}$ & Bulb & Bulb eaten raw (6). & 0.08 \\
\hline $\begin{array}{l}\text { Gagea Iutea (L.) Ker Gawl. } \\
\text { [Liliaceae] }\end{array}$ & - & $\mathrm{H}$ & $\begin{array}{l}\text { Leaf, } \\
\text { Bulb }\end{array}$ & $\begin{array}{l}\text { Leaves are cooked as } \\
\text { vegetables ( } 7) \text {, while bulb is } \\
\text { used as condiment (7). }\end{array}$ & 0.18 \\
\hline $\begin{array}{l}\text { Heracleum candicans Wall. ex } \\
\text { DC. [Apiaceae] }\end{array}$ & Patrala & $\mathrm{H}$ & Root & Raw roots are eaten (5). & 0.06 \\
\hline $\begin{array}{l}\text { Himalaiella heteromalla } \\
\text { (D.Don) Raab-Straube } \\
\text { [Asteraceae] }\end{array}$ & Shublut & $\mathrm{H}$ & Root & Roots eaten raw (6). & 0.08 \\
\hline $\begin{array}{l}\text { Humulus lupulus L. } \\
\text { [Cannabaceae] }\end{array}$ & Hops & $\mathrm{C}$ & Flower & $\begin{array}{l}\text { Flowers are dried and used for } \\
\text { making local wine (13).* }\end{array}$ & 0.17 \\
\hline $\begin{array}{l}\text { Impatiens glandulifera Royle } \\
\text { [Balsaminaceae] }\end{array}$ & Phutong & $\mathrm{S}$ & Seed & Seeds are eaten raw (10). & 0.13 \\
\hline Juglans regia L. [Juglandaceae] & Dun & $\mathrm{T}$ & Fruit & $\begin{array}{l}\text { Kernels edible (28), catkins } \\
\text { cooked as vegetable (12). }\end{array}$ & 0.51 \\
\hline $\begin{array}{l}\text { Koenigia alpina (All.) } \\
\text { T.M.Schust. \& Reveal } \\
\text { [Polygonaceae] }\end{array}$ & Tsokladar & $\mathrm{H}$ & Leaf & $\begin{array}{l}\text { Tender leaves are used as } \\
\text { vegetable (10). }\end{array}$ & 0.13 \\
\hline Lathyrus aphaca L. [Fabaceae] & Jungli matar & $\mathrm{H}$ & $\begin{array}{l}\text { Leaf, } \\
\text { Fruit }\end{array}$ & $\begin{array}{l}\text { Young leaves are eaten as } \\
\text { vegetable (13) and fruits and } \\
\text { seeds are eaten raw (18). }\end{array}$ & 0.41 \\
\hline $\begin{array}{l}\text { Lepidium sativum } \mathrm{L} \text {. } \\
\text { [Brassicaceae] }\end{array}$ & Jungli matar & $\mathrm{H}$ & Leaf & $\begin{array}{l}\text { Young leaves or plant cooked } \\
\text { as vegetable (8). }\end{array}$ & 0.1 \\
\hline $\begin{array}{l}\text { Lepidium virginicum } \mathrm{L} \text {. } \\
\text { [Brassicaceae] }\end{array}$ & Jungli matar & $\mathrm{H}$ & Shoot & $\begin{array}{l}\text { Young shoot occasionally } \\
\text { taken as vegetable (8). }\end{array}$ & 0.1 \\
\hline $\begin{array}{l}\text { Lonicera angustifolia Wall. ex } \\
\text { DC. [Caprifoliaceae] }\end{array}$ & Sagtso & S & Fruit & Ripe fruits are eaten raw (6). & 0.08 \\
\hline $\begin{array}{l}\text { Malva neglecta Wallr. } \\
\text { [Malvaceae] }\end{array}$ & Soc hal & $\mathrm{H}$ & Leaf & $\begin{array}{l}\text { Leaves cooked as vegetable } \\
(28) \text {. }\end{array}$ & 0.36 \\
\hline $\begin{array}{l}\text { Medicago polymorpha } \mathrm{L} \\
\text { [Fabaceae]. }\end{array}$ & Ispit & $\mathrm{H}$ & Leaf & $\begin{array}{l}\text { Tender leaves used as } \\
\text { vegetable (15). }\end{array}$ & 0.19 \\
\hline Medicago sativa L. [Fabaceae] & Ispit & $\mathrm{H}$ & Seed & $\begin{array}{l}\text { Sprouted seeds are eaten as } \\
\text { vegetable (12). }\end{array}$ & 0.15 \\
\hline $\begin{array}{l}\text { Melanoseris lessertiana (DC.) } \\
\text { Decne. [Asteraceae] }\end{array}$ & Gundula & $\mathrm{H}$ & Leaf & $\begin{array}{l}\text { Fresh leaves are eaten as } \\
\text { vegetable after boiling, older } \\
\text { leaves are parboiled (8). }\end{array}$ & 0.1 \\
\hline $\begin{array}{l}\text { Mentha longifolia (L.) L. } \\
\text { [Lamiaceae] }\end{array}$ & Jangli Putna & $\mathrm{H}$ & Leaf & $\begin{array}{l}\text { Leaves used as spice and } \\
\text { condiment (10) and in } \\
\text { preparation of chutney (11). }\end{array}$ & 0.27 \\
\hline Mentha spicata L. [Lamiaceae] & Putna & $\mathrm{H}$ & Leaf & $\begin{array}{l}\text { Leaves used as spice and } \\
\text { condiment (12) and } \\
\text { preparation of chutney (16). }\end{array}$ & 0.36 \\
\hline Morus alba L. [Moraceae] & Tul & $\mathrm{T}$ & Fruit & Ripe fruits are eaten raw (34). & 0.43 \\
\hline
\end{tabular}




\begin{tabular}{|c|c|c|c|c|c|}
\hline Botanical name [Family] & $\begin{array}{l}\text { Local } \\
\text { name }\end{array}$ & Habit & $\begin{array}{l}\text { Part } \\
\text { used }\end{array}$ & Uses & $\mathrm{CI}$ \\
\hline Morus nigra L. [Moraceae] & Tul & $\mathrm{T}$ & Fruit & Fruit is edible (12). & 0.15 \\
\hline $\begin{array}{l}\text { Nasturtium officinale } \\
\text { W.T.Aiton [Brassicaceae] }\end{array}$ & Nagbibir & $\mathrm{H}$ & Leaf & $\begin{array}{l}\text { Leaves are cooked as vegetable } \\
(29) \text {. }\end{array}$ & 0.37 \\
\hline Origanum vulgare L. [Lamiaceae] & $\begin{array}{l}\text { Marzanjosh } \\
\text { / Wanbibir }\end{array}$ & $\mathrm{H}$ & Leaf & $\begin{array}{l}\text { Leaves and young shoot tips } \\
\text { used as spice (14). }\end{array}$ & 0.18 \\
\hline $\begin{array}{l}\text { Orobanche alba Stephan ex } \\
\text { Willd. [Orobanchaceae] }\end{array}$ & Subzgul & $\mathrm{H}$ & Shoot & Plant used as vegetable (11). & 0.14 \\
\hline $\begin{array}{l}\text { Oxalis acetosella L. } \\
\text { [Oxalidaceae] }\end{array}$ & Dangchuch & $\mathrm{H}$ & Leaf & $\begin{array}{l}\text { Leaves eaten raw or added to } \\
\text { cooked food to give astringent } \\
\text { taste (3). }\end{array}$ & 0.04 \\
\hline $\begin{array}{l}\text { Oxalis corniculata L. } \\
\text { [Oxalidaceae] }\end{array}$ & Dangchuch & $\mathrm{H}$ & Leaf & $\begin{array}{l}\text { Leaves eaten raw or added to } \\
\text { cooked food (11). }\end{array}$ & 0.14 \\
\hline $\begin{array}{l}\text { Oxyria digyna (L.) Hill } \\
\text { [Polygonaceae] }\end{array}$ & Shoop & $\mathrm{H}$ & Leaf & $\begin{array}{l}\text { Leaves pleasantly sour in taste } \\
\text { and refreshing are eaten raw } \\
\text { (8) and also used to make } \\
\text { chutney (8). }\end{array}$ & 0.21 \\
\hline $\begin{array}{l}\text { Phytolacca acinosa Roxb. } \\
\text { [Phytolaccaceae] }\end{array}$ & Arail & $\mathrm{H}$ & Leaf & $\begin{array}{l}\text { Leaves are cooked as vegetable } \\
\text { (5). }\end{array}$ & 0.06 \\
\hline $\begin{array}{l}\text { Pinus gerardiana Wall. ex } \\
\text { D.Don [Pinaceae] }\end{array}$ & Fita & $\mathrm{T}$ & Seed & Seeds are edible (35). & 0.45 \\
\hline $\begin{array}{l}\text { Plantago lanceolata L. } \\
\text { [Plantaginaceae] }\end{array}$ & Gul & $\mathrm{H}$ & Leaf & $\begin{array}{l}\text { Tender leaves are used as } \\
\text { vegetable (15). }\end{array}$ & 0.19 \\
\hline $\begin{array}{l}\text { Podophyllum hexandrum } \\
\text { Royle [Berberidaceae] }\end{array}$ & Bankakdi & $\mathrm{H}$ & Fruit & $\begin{array}{l}\text { Ripe fruits are eaten raw by } \\
\text { shepherds (11). }\end{array}$ & 0.14 \\
\hline $\begin{array}{l}\text { Polygonum aviculare L. } \\
\text { [Polygonaceae] }\end{array}$ & Endrani & $\mathrm{H}$ & Leaf & $\begin{array}{l}\text { Tender leaves are used as } \\
\text { vegetable (9). }\end{array}$ & 0.12 \\
\hline $\begin{array}{l}\text { Potentilla indica (Andrews) } \\
\text { Th.Wolf [Rosaceae] }\end{array}$ & $\begin{array}{l}\text { Sarpingdac } \\
\mathrm{h}\end{array}$ & $\mathrm{H}$ & Fruit & Fruit is edible (18). & 0.23 \\
\hline $\begin{array}{l}\text { Prunus armeniaca L. } \\
\text { [Rosaceae] }\end{array}$ & Cheir & $\mathrm{T}$ & Fruit & Fruits are edible (31). & 0.4 \\
\hline $\begin{array}{l}\text { Prunus cornuta (Wall. ex Royle) } \\
\text { Steud. [Rosaceae] }\end{array}$ & Zamb & $\mathrm{T}$ & Fruit & Fruits are edible (26). & 0.33 \\
\hline $\begin{array}{l}\text { Punica granatum L. } \\
\text { [Lythraceae] }\end{array}$ & Dan & $\mathrm{T}$ & Fruit & $\begin{array}{l}\text { Fruit is edible and used to } \\
\text { make chutney (27). }\end{array}$ & 0.35 \\
\hline $\begin{array}{l}\text { Pyrus paschia Buch.-Ham. ex } \\
\text { D.Don [Rosaceae] }\end{array}$ & Naakh & $\mathrm{T}$ & Fruit & Fruits edible (26). & 0.33 \\
\hline $\begin{array}{l}\text { Rheum australe D. Don } \\
\text { [Polygonaceae] }\end{array}$ & Lachu & $\mathrm{H}$ & Shoot & $\begin{array}{l}\text { Young shoots are cooked as } \\
\text { vegetables (24) and also used } \\
\text { for making chutney ( } 7) \text {. }\end{array}$ & 0.4 \\
\hline $\begin{array}{l}\text { Rheum webbianum Royle } \\
\text { [Polygonaceae] }\end{array}$ & Lachu & $\mathrm{H}$ & Shoot & $\begin{array}{l}\text { Young shoots are cooked as } \\
\text { vegetables (17) and also used } \\
\text { for making chutney (5). }\end{array}$ & 0.28 \\
\hline $\begin{array}{l}\text { Rhododendron arboreum } \\
\text { Sm. [Ericaceae] }\end{array}$ & Pragu & $\mathrm{S}$ & Flower & $\begin{array}{l}\text { Fresh flowers are eaten raw (7) } \\
\text { and juice is also extracted from } \\
\text { them (16). }\end{array}$ & 0.29 \\
\hline $\begin{array}{l}\text { Rhododendron } \\
\text { campanulatum D.Don } \\
\text { [Ericaceae] }\end{array}$ & Burshu & $\mathrm{S}$ & Flower & $\begin{array}{l}\text { Flowers are used for making } \\
\text { chutney (7) \& juices }(7) \text {. }\end{array}$ & 0.18 \\
\hline
\end{tabular}


Sajan Thakur \& Harish Chander Dutt 285

\begin{tabular}{|c|c|c|c|c|c|}
\hline Botanical name [Family] & $\begin{array}{l}\text { Local } \\
\text { name }\end{array}$ & Habit & $\begin{array}{l}\text { Part } \\
\text { used }\end{array}$ & Uses & $\mathbf{C I}$ \\
\hline $\begin{array}{l}\text { Robinia pseudoacacia L. } \\
\text { [Fabaceae] }\end{array}$ & Robinia & $\mathrm{T}$ & Flower & $\begin{array}{l}\text { Floral buds are boiled, } \\
\text { squeezed and fried to make } \\
\text { vegetable (5). }\end{array}$ & 0.06 \\
\hline $\begin{array}{l}\text { Rosa webbiana Wall. ex Royle } \\
\text { [Rosaceae] }\end{array}$ & Jungli gulab & S & Flower & $\begin{array}{l}\text { Petals used in making drinks } \\
\text { (13). }\end{array}$ & 0.17 \\
\hline $\begin{array}{l}\text { Rubus biflorus Buch.-Ham. ex } \\
\text { Sm. [Rosaceae] }\end{array}$ & Onchh & S & Fruit & $\begin{array}{l}\text { Sweet fruits are eaten raw } \\
\text { when ripe (12). }\end{array}$ & 0.15 \\
\hline $\begin{array}{l}\text { Rubus ellipticus } \mathrm{Sm} . \\
\text { [Rosaceae] }\end{array}$ & Onchh & $S$ & Fruit & Fruits are eaten raw (28). & 0.36 \\
\hline Rubus fruticosus L. [Rosaceae] & Onchh & $\mathrm{S}$ & Fruit & Sweet fruits are eaten raw (7). & 0.09 \\
\hline $\begin{array}{l}\text { Rubus hoffmeisterianus Kunth } \\
\text { \& C.D.Bouche [Rosaceae] }\end{array}$ & Onchh & $S$ & Fruit & Fruits are eaten raw (7). & 0.09 \\
\hline $\begin{array}{l}\text { Rubus niveus Thunb. } \\
\text { [Rosaceae] }\end{array}$ & Onchh & $S$ & Fruit & $\begin{array}{l}\text { Fruits are eaten raw when ripe } \\
\text { (15). }\end{array}$ & 0.19 \\
\hline $\begin{array}{l}\text { Rubus pedunculosus D.Don } \\
\text { [Rosaceae] }\end{array}$ & Onchh & S & Fruit & $\begin{array}{l}\text { Sweet fruits are eaten raw } \\
\text { when ripe (3). }\end{array}$ & 0.04 \\
\hline $\begin{array}{l}\text { Rumex acetosa L. } \\
\text { [Polygonaceae] }\end{array}$ & Tsoktsin & $\mathrm{H}$ & Leaf & $\begin{array}{l}\text { Leaves are cooked as vegetable } \\
\text { (9). }\end{array}$ & 0.12 \\
\hline $\begin{array}{l}\text { Rumex dentatus L. } \\
\text { [Polygonaceae] }\end{array}$ & Ambli & $\mathrm{H}$ & Leaf & $\begin{array}{l}\text { Leaves are cooked as vegetable } \\
\text { (8). }\end{array}$ & 0.1 \\
\hline $\begin{array}{l}\text { Rumex nepalensis Spreng. } \\
\text { [Polygonaceae] }\end{array}$ & Habul & $\mathrm{H}$ & Leaf & $\begin{array}{l}\text { Leaves are cooked as vegetable } \\
\text { (6). }\end{array}$ & 0.08 \\
\hline $\begin{array}{l}\text { Silene baccifera (L.) Durande } \\
\text { [Caryophyllaceae] }\end{array}$ & Shelich & $\mathrm{H}$ & Leaf & $\begin{array}{l}\text { Tender leaves are cooked and } \\
\text { eaten with rice (6). }\end{array}$ & 0.08 \\
\hline $\begin{array}{l}\text { Solanum nigrum } \mathrm{L} \text {. } \\
\text { [Solanaceae] }\end{array}$ & Kambai & $\mathrm{H}$ & Fruit & Fruit is edible (10). & 0.13 \\
\hline $\begin{array}{l}\text { Sonchus asper (L.) Hill } \\
\text { [Asteraceae] }\end{array}$ & Dudhand & $\mathrm{H}$ & Leaf & $\begin{array}{l}\text { Leaves are cooked as vegetable } \\
\text { (5). }\end{array}$ & 0.06 \\
\hline $\begin{array}{l}\text { Taraxacum officinale } \\
\text { F.H.Wigg. [Asteraceae] }\end{array}$ & Hand & $\mathrm{H}$ & Leaf & $\begin{array}{l}\text { Leaves are cooked as vegetable } \\
\text { (8). }\end{array}$ & 0.1 \\
\hline $\begin{array}{l}\text { Taxus wallichiana Zucc. } \\
\text { [Taxaceae] }\end{array}$ & Pustil & $\mathrm{T}$ & Bark & $\begin{array}{l}\text { Bark is used as substitute for } \\
\text { tea (17). }\end{array}$ & 0.22 \\
\hline $\begin{array}{l}\text { Thymus linearis Benth. } \\
\text { [Lamiaceae] }\end{array}$ & Piplichi & $\mathrm{H}$ & Leaf & $\begin{array}{l}\text { Fresh leaves are used for } \\
\text { making vegetables (15). }\end{array}$ & 0.19 \\
\hline $\begin{array}{l}\text { Thymus mongolicus (Roniger) } \\
\text { Roniger [Lamiaceae] }\end{array}$ & Ban-ajwain & $\mathrm{H}$ & Shoot & $\begin{array}{l}\text { Aerial parts are used as spice } \\
\text { (5). }\end{array}$ & 0.06 \\
\hline Trifolium repens $\mathrm{L} . \quad$ [Fabaceae] & Chopati & $\mathrm{H}$ & Shoot & Used as vegetable (13). & 0.17 \\
\hline $\begin{array}{l}\text { Trillium govanianum Wall. ex } \\
\text { D.Don [Melanthiaceae] }\end{array}$ & Nagchattri & $\mathrm{H}$ & Leaf & $\begin{array}{l}\text { Leaves cooked as vegetable } \\
\text { (15). }\end{array}$ & 0.19 \\
\hline $\begin{array}{l}\text { Tulipa clusianai Redoute } \\
\text { [Liliaceae] }\end{array}$ & - & $\mathrm{H}$ & Tuber & Tubers edible (6). & 0.08 \\
\hline $\begin{array}{l}\text { Turritis glabra L. } \\
\text { [Brassicaceae] }\end{array}$ & - & $\mathrm{H}$ & Leaf & $\begin{array}{l}\text { Tender leaves are used to } \\
\text { make vegetables (4). }\end{array}$ & 0.05 \\
\hline $\begin{array}{l}\text { Typha domingensis Pers. } \\
\text { [Typhaceae] }\end{array}$ & Petz & $\mathrm{H}$ & Tuber & $\begin{array}{l}\text { Rhizome is eaten as vegetable } \\
\text { (6). }\end{array}$ & 0.08 \\
\hline Urtica dioica L. [Urticaceae] & Soy & $\mathrm{H}$ & Leaf & $\begin{array}{l}\text { Leaves are boiled in water for } \\
\text { about ten minutes and then } \\
\text { cooked with salt and chili } \\
\text { powder (18). }\end{array}$ & 0.23 \\
\hline
\end{tabular}


Traditional knowledge in Himalayan Kishtwar district

\begin{tabular}{|l|l|l|l|l|l|}
\hline Botanical name [Family] & $\begin{array}{l}\text { Local } \\
\text { name }\end{array}$ & Habit & $\begin{array}{l}\text { Part } \\
\text { used }\end{array}$ & Uses \\
\hline $\begin{array}{l}\text { Viburnum grandiflorum Wall. } \\
\text { ex DC. [Viburnaceae] }\end{array}$ & Kullam & S & Fruit & Fruit is edible (22). & 0.28 \\
\hline $\begin{array}{l}\text { Vicia faba L. [Fabaceae] } \\
\text { Jungli matar }\end{array}$ & H & Seed & $\begin{array}{l}\text { Seeds and fruits are edible } \\
(15) .\end{array}$ & 0.19 \\
\hline $\begin{array}{l}\text { Zanthoxylum armatum DC. } \\
\text { Rutaceae] }\end{array}$ & Timbru & S & Fruit & $\begin{array}{l}\text { Fruits are used to make } \\
\text { chutney (16). }\end{array}$ & 0.21 \\
\hline $\begin{array}{l}\text { Ziziphus jujuba Mill. } \\
\text { Rhamnaceae] }\end{array}$ & Bair & S & Fruit & Fruits eaten raw (27). & 0.35 \\
\hline $\begin{array}{l}\text { Ziziphus oxyphylla Edgew. } \\
\text { Rhamnaceae] }\end{array}$ & Bair & S & Fruit & Fruit is edible (22). & 0.28 \\
\hline
\end{tabular}

$\mathrm{H}=$ Herb; $\mathrm{S}=$ Shrub; $\mathrm{T}=$ Tree and $\mathrm{C}=$ Climber.

$*=$ new records on local traditional uses

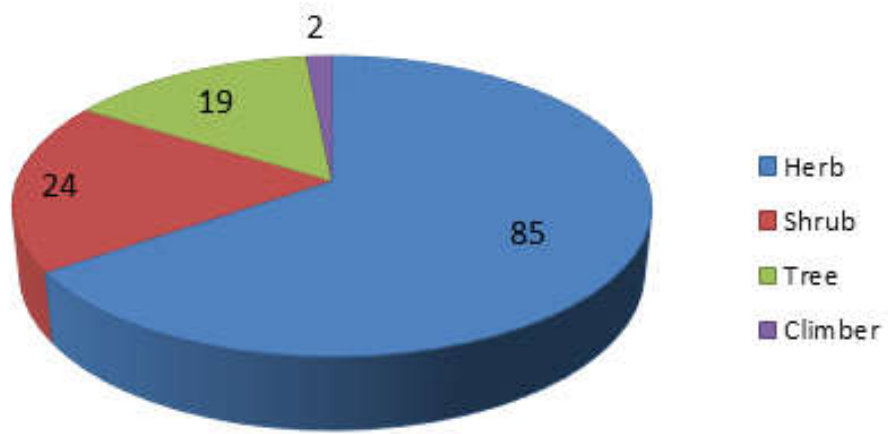

Figure 2. Habit-groups of wild edible plants of district Kishtwar

Table 2. Use reports and Factor informant consensus (Fic) of various categories of wild edible plants of district Kishtwar

\begin{tabular}{clccc}
\hline S. No. & \multicolumn{1}{c}{ Category } & $\begin{array}{c}\text { Number of use } \\
\text { reports (Nur) }\end{array}$ & $\begin{array}{c}\text { Number of taxa } \\
\text { (Nt) }\end{array}$ & Fic \\
\hline 1. & Bread and pudding & 39 & 3 & 0.95 \\
$\mathbf{2 .}$ & Vegetables & 734 & 56 & 0.92 \\
$\mathbf{3 .}$ & Spice and Condiments & 246 & 15 & 0.94 \\
$\mathbf{4 .}$ & Beverages & 146 & 12 & 0.92 \\
$\mathbf{5 .}$ & Soup & 32 & 2 & 0.97 \\
$\mathbf{6 .}$ & Raw & 814 & 51 & 0.94 \\
$\mathbf{7 .}$ & Fritters & 37 & 3 & 0.94 \\
$\mathbf{8 .}$ & Chutney & 97 & 8 & 0.93 \\
\hline
\end{tabular}

Ferula jaeschkeana was observed to be most important wild edible plant species in the study area with high cultural importance index $(\mathrm{CI}=0.64)$ followed by Elwendia persica and Diplazium esculenta $(\mathrm{CI}=0.59 \mathrm{each})$ and Berberis lycium and Juglans regia $(\mathrm{CI}=0.51$ each $)($ Table 1$)$. The species with maximum CI values are most used plant species as wild edible plant in the study area. Among the documented wild edible plants 56 were cooked as vegetable, 51 were eaten raw, 15 were used as spice and condiments and 12 were used to prepare beverages (local 


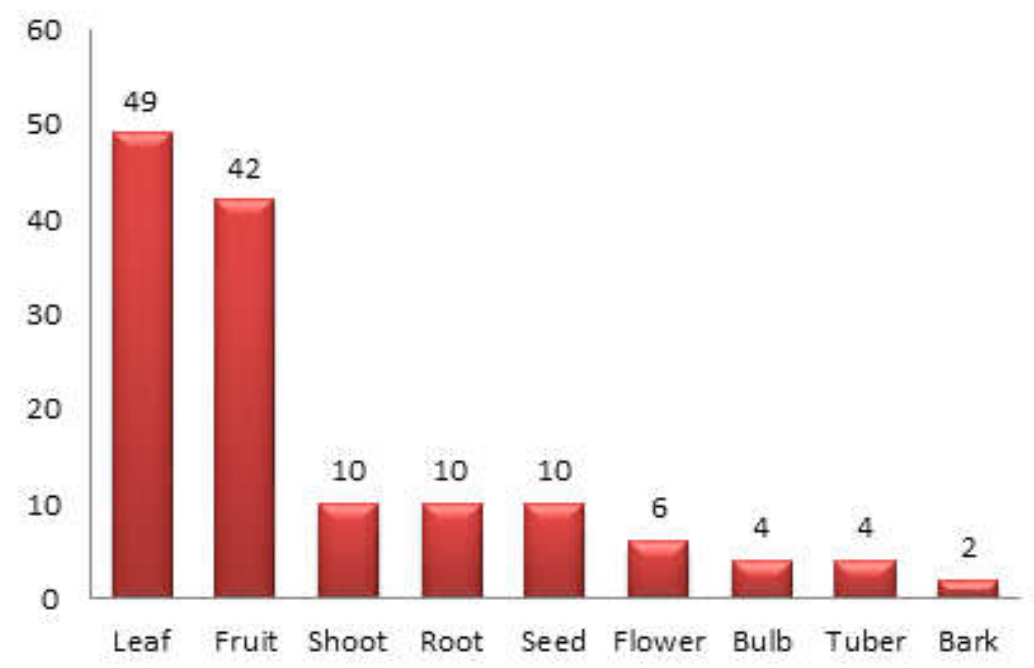

Figure 3. Plant parts of wild edible plants of district Kishtwar

wine, tea and juices). Factor informant consensus (Fic) was greater than 0.90 for all the categories and which determine the maximum homogeneity of knowledge about the usage of wild edible plant species in the study area (Table 2).

\section{DISCUSSION}

As major population of our country India resides in villages and far-flung areas, so wild edible plants are the noteworthy source of food in these areas as a source of nutrition. Women folks are the main nutrition provider of maximum of the village families. In the present study females $(57.7 \%)$ possessed maximum knowledge of wild edible plants as compared to males $(42.3 \%)$. The females of the villages during every collect different wild edible plant species which are available during different seasons. They possess more knowledge about wild edible plants as compared to male folks as they are directly associated with the household and food preparation activities (Junsongduang et al. 2014). Singh et al. (2016) have reported 111 wild edible plants from Kashmir Himalaya whereas Thakur et al. (2017) have recorded 50 plant species from tribal areas of Western Himalaya as wild edible plants. Bhatia et al. (2018) have reported 90 species from Udhampur district of Jammu and Kashmir whereas, in present study 130 plant species are reported as wild edible foods from the district Kishtwar of UT of J\&K. In study area Rosaceae is the most dominant family (Thakur et al. 2017; Singh et al. 2016; Menendez-Baceta et al. 2015; Geng et al. 2016). Maximum 56 plant species in the present study are cooked as vegetable and also have maximum CI values. Thakur et al. (2017), Misra et al. (2008), Singh et al. (2016) and Kumar and Hamal (2009) have also reported maximum vegetables as the source of wild food with higher CI values.

A total of 6 species (Oxyria digyna, Punica granatum, Rheum australe, Rheum webbianum, Rhododendron campanulatum and Zanthoxylum armatum) in the study area are used in the preparation of chutney which is ready to eat food for instant consumption. Chutney is a part of daily meal in the study area especially during summer season as it is a very good appetizer, antigastric, antispasmodic and improves digestion (Rao et al. 2015; Bhatia et al. 2014). Owing to aromatic properties of Carum carvi, Elwendia persica, Ferula jaeschkeana, Mentha longifolia, Mentha spicata, Origanum vulgare and Thymus mongolicus are added to vegetables and pulses as a source of spice and condiments in district Kishtwar. Majority of these spices like Elwendia persica, Carum carvi, 
Mentha longifolia and Mentha spicata are used to cure stomachache, indigestion and other digestive problems (Thakur \& Dutt 2019; Saini \& Reddy 2013; Thakur et al. 2020b).

The local people in district Kishtwar possess rich traditional knowledge about the use of plants as a source of medicine especially against gastrointestinal ailments (Thakur et al. 2020b). Likewise, in present species the Fic values greater than 0.92 revealed once again that there is good homogeneity of knowledge among the local people in district Kishtwar about the use of wild plants as a source of food.

\section{CONCLUSION}

The current study revealed that the oral traditional knowledge about the use of wild edible plants is still in practice among the local populace of district Kishtwar. Rich plant diversity as a source of wild food with greater categorical consensus for their local usage also supports this account. The people in the study area are dependent on the wild resources not only for their food and nutrition but also for income generation. These plant species are greatly used in the Himalayan region which is an outcome of the occurrence of similar traditions and cultures in the region. Out of 130 plant species reported as edible ones eight species i.e., Aesculus indica, Arisaema propinquum, Arnebia euchroma, Arnebia guttata, Betula utilis, Daphne gnidioides, Dioscorea deltoidea and Humulus lupulus are exclusively used for food and drink purposes by the people of Kishtwar district and are reported first time. The current study also reveals that sap of Ferula jaeschkeana, seeds of Elwendia persica and fronds of Diplazium esculenta have significant cultural importance index in the region and consumed on regular basis. Further, all the dietary preparations made by the people using 130 plant species have shown high factor informant consensus between 0.92 to 0.97 , which determines the homogeneity of traditional knowledge on usage of wild edible plants among the informants in the region. High value of this index also determines the undeviating transfer of the oral traditional knowledge from generation to generation in Kishtwar district. The persistence of knowledge for the consumption of conventional foods is a powerful tool in the conservation of ethnic identity and cultures and plant diversity in one or the other way. So, it is very much important that national and international authorities recognize the contribution of rural communities to the diversification of traditional knowledge on human nutrition. Higher authorities need to work in collaboration with local folk for the reappraisal of folk knowledge on wild edible plants which will be helpful in eradication poverty from global level.

\section{Acknowledgements}

Authors are highly thankful to Head, Department of Botany, University of Jammu and Convener, University Grants Commission-Special Assistance Program (DRS-II) for providing necessary facilities to carry out field visits and consultation of Herbarium.

\section{LITERATURE CITED}

Beluhan, S. \& Ranogajec, A. 2010. Chemical composition and non-volatile components of Crotial wild edible mushrooms. Food Chemistry 124: 1076 - 1082.

Bhatia, H.; Sharma, Y.P.; Manhas, R.K. \& Kumar, K. 2014. Ethnomedicinal plants used by the villagers of district Udhampur, J\&K, India. Journal of Ethnopharmacology 151(2): 1005 1018.

Bhatia, H.; Sharma, Y.P.; Manhas, R.K. \& Kumar, K. 2015. Traditional phytoremedies for the treatment of menstrual disorders in district Udhampur, J\&K, India. Jounal of Ethnopharmacology 160: 202 - 210. 
Bhatia, H.; Sharma, Y.P.; Manhas, R.K. \& Kumar, K. 2018. Traditionally used wild edible plants of district Udhampur, J\&K, India. Journal of Ethnobiology and Ethnomedicine 14(73). DOI:https://doi.org/10.1186/s13002-018-0272-1.

Binu, S. 2010. Wild edible plants used by the tribals in Pathanamthitta district, Kerala. Indian Journal of Traditional Knowledge 9: 309 - 312.

Burlingame, B. 2000. Wild nutrition. Journal of Food Composition and Analysis 13: 99 - 100.

Gazzaneo, L.R.S.; Lucena, R.F.P. \& Albuquerque, U.P. 2005. Knowledge and use of medicinal plants by local specialists in a region of Atlantic Forest in the state of Pernambuco (northeastern Brazil). Journal of Ethnobiology and Ethnomedicine doi:101186/1746-4269-1-9.

Geng, Y.; Zhang, Y.; Ranjitkar, S.; Huai, H. \& Wang, Y. 2016. Traditional knowledge and its transmission of wild edibles used by the Naxi in Baidi Village, northwest Yunnan province. Journal of Ethnobiology and Ethnomedicine 12: 10.

Grivetti, L.E. \& Ogle, B.M. 2000. Value of traditional foods in meeting macro- and micro nutrient needs: the wild plant connection. Nutrition Research Reviews 13(1): 31 - 46.

Haridasan, K., L.R. Bhuyan \& Deori, M. 1990. Wild edible plants of Arunachal Pradesh. Arunachal forest News 8(1\&2): 7.

Heinrich, M.; Ankli, A.; Frei, B.; Weimann, C. \& Sticher, O. 1998. Medicinal plants in Mexico: healers' consensus and cultural importance. Social Science and Medicine 47: 1863 - 1875.

Hussain, J.; Khan, A.L.; Rehman, N.; Hamayun, M.; Shah, T.; Nisar, M.; Bano, T.; Shinwari, Z.K. \& Lee, I. 2009. Proximate and nutrient analysis of selected vegetable species: a case study of Karak region, Pakistan. African Journal of Biotechnology 8(12): 2725 - 2729.

Jain, S.K. 1964. Wild plants foods of the tribes of Bastar (Madhya Pradesh). Proceedings National Institute of Sciences, India 30B (2): 56 - 80.

Junsongduang, A.; Balslev, H.; Inta, A.; Jampeetong, A. and Wangpakapattanawong, P. 2014. Karen and Lawa medicinal plant use: uniformity or ethnic divergence? Journal of Ethnopharmacology 151: 517 - 527.

Kayang, H. 2007. Tribal knowledge on wild edible plants of Meghalaya, Norteast India. Indian Journal of Traditional Knowledge 6: 177 - 181.

Kulkarni, D.K.; Agte, V.V. \& Kumbhojkar, M.S. 2003. Leafy vegetables consumed by Mahadeokoli tribe in Western Maharashtra with their nutritional potential. Ethnobotany 15: $34-38$.

Kumar, S. \& Hamal, I.A. 2009. Wild edibles of Kishtwar high altitude national park in northwest Himalaya, Jammu and Kashmir (India). Ethnobotanical Leaflets 13: 195 - 202.

Kumer, V. 2003. Wild edible plants of Surguja District of Chattisgarh state, India. Journal of Economic and Taxonomic Botany 27(2): $272-282$.

Luczaj, L.; Koncic, M.Z.; Milicevic, T.; Dolina, K. \& Pandza, M. 2013. Wild vegetable mixes sold in markets of Dalmatia (southern Croatia). Jornal of Ethnobiology and Ethnomedicine 9(1): 2.

Menendez-Baceta, G.; Aceituno-Mata, L.; Reyes-García, V.; Tardío, J.; Salpeteur, M. \& Pardode-Santayana, M. 2015. The importance of cultural factors in the distribution of medicinal plant knowledge: a case study in four Basque regions. Journal of Ethnopharmacology 161: $116-127$.

Misra, S.; Maikhuri, R.K.; Kala, C.P.; Rao, K.S. \& Saxena, K.G. 2008. Wild leafy vegetables: a study of their subsistence dietetic support to the inhabitants of Nanda Devi biosphere reserve India. Journal of Ethnobiology and Ethnomedicine 4: 15. 
Mohan Ram, H.Y. 2000. Plant Resources of Indian Himalaya. 9th G P Pant Memorial Lecture, G B Pant Institute of Himalayan Development. Gangtok: Sikkim.

Negi, K.S. 1988. Some little known Wild edible plants of UP Hills. J. Econ. Taxon. Bot., 12(2): $245-360$.

Pandita, S. \& Dutt, H.C., 2018. Wild edible plants as invaluable ethnomedicine among mountainous people of Bhaderwah, Jammu and Kashmir, India. Journal of Traditional and Folk Practices 06(2): $25-38$.

Pundir, Y.P.S. \& Singh, D. 2002. Ethnobotanical wild food plants of Jaunsar-Bawar (Western Himalaya), Uttaranchal. Indian Forester 128(5): 571 - 582.

Rao, P.K.; Hasan, S.S.; Bhellum, B.L. \& Manhas, R.K. 2015. Ethnomedicinal plants of Kathua district, J\&K, India. Journal of Ethnopharmacology 171: 12 - 27.

Rashid, A.; Anand, V.K. and Serwer, J. 2008. Less known wild edible plants used by the Gujjar tribe of district Rajouri, Jammu and Kashmir state - India. International Journal of Botany 4(2): 219-24.

Rathore, M. 2009. Nutrient content of important fruit trees from arid zone of Rajasthan. Journal of Horticulture and Forestry 1: 103.

Reyes-Garcia, V.; Menendez-Baceta, G.; Aceituno-Mata, L.; Acosta-Naranjo, R.; Calvet-Mir, L.; Dominguez, P. \& Rodriguez-Franco, R. 2015. From famine food to delicatessen: interpreting trends in the use of wild edible plants through cultural ecosystem services. Ecological Economics 120: 303 - 311.

Saini, S.C. \& Reddy, G.B.S. 2013. Murraya koenigii. Journal of Pharmacy and Biological Sciences 7(6): $15-18$.

Schippmann, U.; Cunningham, A.B. \& Leaman, D.J. 2002. Impact of cultivation and gathering of medicinal plants on biodiversity: global trends and issues. In: Biodiversity and the ecosystem approach in agriculture, forestry and fisheries. Rome: FAO.

Sharma, B.M. and Kachroo, P. 1983. Flora of Jammu and plants of neighbourbood. Dehradun, India: Bishen Singh Mahender Pal Singh.

Sharma, R.; Manhas, R.K. \& Magotra, R. 2012. Ethnoveterinary remedies of diseases among Milk yielding animals in Kathua, Jammu and Kashmir, India. Journal of Ethnopharmacology 141(1): $265-272$.

Singh, B.; Sultan, P.; Hassan, Q.P.; Gairola, S. \& Bedi, Y.S. 2016. Ethnobotany, traditional knowledge and diversity of wild edible plants and fungi: a case study in the Bandipora District of Kashmir Himalaya, India. Journal of Herbs, Spices and Medicinal Plants 22(3): $247-278$.

Singh, J.; Rajasekaran, A.; Negi, A.K. \& Negi, S.P. 2019. Utilization of edible wild plants as supplementary source of nutrition by the indigenous communities in Kinnaur district, Himachal Pradesh, India. Indian Forester 145(6): 561 - 577.

Sundriyal, M., Sundriyal, R.C. and E. Sharma 2004. Dietary use of wild plant resources in the Sikkim, Himalaya, India. Economic Botany 58: 626 - 638.

Swami, A. \& Gupta, B.K. 1998. Flora of Udhampur. Dehradun, India: Bishen Singh Mahendra Pal Singh.

Tardio, J. \& Pardo-de-Santayana, M. 2008. Cultural importance indices: a comparative analysis based on the useful wild plants of southern Cantabria (northern Spain). Economic Botany 62: $24-39$. 
Thakur, A.; Singh, S. \& Puri, S. 2020a. Exploration of wild edible plants used as food by Gaddis-A tribal community of the Western Himalaya. The Scientific World Journal DOI:https://doi.org/10.1155/2020/6280153.

Thakur, D.; Sharma, A. \& Uniyal, S.K. 2017. Why they eat, what they eat: patterns of wild edible plants consumption in a tribal area of Western Himalaya. Journal of Ethnobiology and Ethnomedicine 13: 70.

Thakur, S. \& Dutt, H.C. 2019. Bunium persicum (Boiss.) Fedtsch. Distribution, Botany and Agrotechnology. In: Plants of Commercial Values (Ed. Bikarma Singh). Pp. 89 - 95.

Thakur, S.; Tashi, N.; Singh, B.; Dutt, H.C. \& Singh, B. 2020b. Ethnobotanical plants used for gastrointestinal ailments by the inhabitants of Kishtwar plateau in Northwestern Himalaya, India. Indian Journal of Traditional Knowledge 19(2): 288 - 298.

Vizhi, M.M. \& Lohidas, J. 2020.Studies on wild edible plants consumed by the tribes of Kanyakumari wildlife sanctuary, India. Plant Archives 20 (2): 6503 - 6509. 